\title{
Experimental comparison of grating- and propagation-based hard X-ray phase tomography of soft tissue
}

\author{
S. Lang, ${ }^{1}$ I. Zanette, ${ }^{2,3, a)}$ M. Dominietto, ${ }^{1,4}$ M. Langer, ${ }^{3,5}$ A. Rack, ${ }^{3}$ G. Schulz, ${ }^{1}$ G. Le Duc, ${ }^{3}$ \\ C. David, ${ }^{6}$ J. Mohr, ${ }^{7}$ F. Pfeiffer, ${ }^{2}$ B. Müller, ${ }^{1}$ and T. Weitkamp ${ }^{3,8}$ \\ ${ }^{1}$ Biomaterials Science Center, University of Basel, Basel, Switzerland \\ ${ }^{2}$ Physik-Department und Institut für Medizintechnik, Technische Universität München, Garching, Germany \\ ${ }^{3}$ European Synchrotron Radiation Facility, Grenoble, France \\ ${ }^{4}$ Institute for Biomedical Engineering, ETH Zürich, Zürich, Switzerland \\ ${ }^{5}$ Université de Lyon, CREATIS; CNRS UMR5220; Inserm U1044; INSA-LYON; Université de Lyon 1, \\ Villeurbane, France \\ ${ }^{6}$ Laboratory for Micro- and Nanotechnology, Paul Scherrer Institut, Villigen, Switzerland \\ ${ }^{7}$ Institute of Microstructure Technology, Karlsruhe Institute of Technology, Eggenstein-Leopoldshafen, \\ Germany \\ ${ }^{8}$ Synchrotron Soleil, Gif-sur-Yvette, France
}

(Received 27 April 2014; accepted 24 September 2014; published online 21 October 2014)

\begin{abstract}
When imaging soft tissues with hard X-rays, phase contrast is often preferred over conventional attenuation contrast due its superior sensitivity. However, it is unclear which of the numerous phase tomography methods yields the optimized results at given experimental conditions. Therefore, we quantitatively compared the three phase tomography methods implemented at the beamline ID19 of the European Synchrotron Radiation Facility: X-ray grating interferometry (XGI), and propagation-based phase tomography, i.e., single-distance phase retrieval (SDPR) and holotomography (HT), using cancerous tissue from a mouse model and an entire heart of a rat. We show that for both specimens, the spatial resolution derived from the characteristic morphological features is about a factor of two better for HT and SDPR compared to XGI, whereas the XGI data generally exhibit much better contrast-to-noise ratios for the anatomical features. Moreover, XGI excels in fidelity of the density measurements, and is also more robust against low-frequency artifacts than HT, but it might suffer from phase-wrapping artifacts. Thus, we can regard the three phase tomography methods discussed as complementary. The application will decide which spatial and density resolutions are desired, for the imaging task and dose requirements, and, in addition, the applicant must choose between the complexity of the experimental setup and the one of data processing. (C) 2014 AIP Publishing LLC. [http://dx.doi.org/10.1063/1.4897225]
\end{abstract}

\section{INTRODUCTION}

The common method for microscopic investigation of soft tissues of human and animal origin is histology. Visiblelight microscopy of histological sections reaches submicrometer lateral spatial resolution but the sample has to be cut irreversibly into micrometer-thick slices, which essentially restricts sectioning to $2 \mathrm{D}$ investigations. In order to obtain the 3D morphology, serial sectioning can be applied, which requires, however, the combination of the soft tissue slices in an appropriate manner; see, for example, Refs. 1 and 2. Furthermore, it usually requires invasive preparation procedures that involve fixation, dehydration or freezing, and embedding. These time-consuming processes often cause local stress and strain within the inhomogeneous soft tissues. $^{3}$ The related local deformations can be corrected by means of less detailed X-ray micro computed tomography $(\mu \mathrm{CT})$ data. True micrometer resolution $\mu \mathrm{CT}$, however, is incompatible with in-vivo studies because of the high X-ray dose deposited in the investigated object. Nonetheless, on extracted tissue, often termed biopsy, it yields highresolution $3 \mathrm{D}$ data of the specimen morphology.
Unfortunately, the composition and density variations in soft tissue specimens only result in weak X-ray absorption contrast. Contrast-enhancing techniques like staining or corrosion casts can be used, ${ }^{4}$ but there are tissues for which these techniques are known to fail, e.g., tumors in the final stage. , $6^{2}$

An alternative to X-ray absorption contrast in $\mu \mathrm{CT}$ studies of soft tissues is phase contrast, in which the image signal is generated by distortions in the wavefront phase caused by the specimen. Common phase contrast methods include crystal interferometry, analyzer-based imaging, propagationbased imaging (PBI), and, more recently, grating-based methods. $^{7-11}$ These methods use dedicated experimental setups and provide characteristic signals, but they have in common that the signal in the raw images is related to variations in the X-ray refractive index within the object under study. Absorption and refraction of $\mathrm{X}$ rays of a given wavelength $\lambda$ in a given material can be described by means of the local complex refractive index $n$, which is defined as

$$
n(x, y, z)=1-\delta(x, y, z)+i \beta(x, y, z) .
$$

Here, the decrement $\delta$ describes refraction, whereas the imaginary part $\beta$ describes attenuation. Absorption

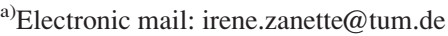


tomography usually yields the three-dimensional distribution of the linear attenuation coefficient $\mu$ which is proportional to $\beta: \mu=4 \pi \beta / \lambda$.

For hard $\mathrm{X}$ rays and for all materials, $\delta$ and $\beta$ are many orders of magnitude smaller than unity, which reflects the weak refraction and attenuation of $\mathrm{X}$ rays. For $\mathrm{X}$-ray photon energies above $10 \mathrm{keV}$, however, the values of $\delta$ for light materials, such as water and soft tissue, are several orders of magnitude higher than the values of $\beta$. Using phase contrast can therefore improve the contrast-to-noise ratio.

The image signal in X-ray phase contrast, however, is generally not related to $\delta$ as simply as the absorptioncontrast signal is to $\beta$. Tomographic retrieval of $\delta(x, y, z)$ from phase-contrast images therefore requires an additional data processing step: the reconstruction of the wavefront phase profile $\varphi(x, y)$ from the images recorded by the detector (phase retrieval). The phase shift induced by the object can be written as

$$
\varphi(x, y)=\frac{2 \pi}{\lambda} \int \delta(x, y, z) d z .
$$

Equation (2) shows that $\varphi$ is the projection of $\delta$ onto the $(x, y)$ plane perpendicular to the optical axis. Therefore, its tomographic reconstruction yields the spatial distribution $\delta(x, y, z)$. The phase retrieval algorithm strongly depends on the technique used to record the phase-contrast images, and, for the same techniques, on the approximations used to model the experiment. In this paper, the term phase tomography refers to the combined processes of data collection, phase retrieval, and tomographic reconstruction, or its results.

In order to identify the appropriate phase tomography approach for visualization and quantification of microstructures in soft condensed matter, such as anatomical features within animal and human tissue, a detailed comparison of the spatial and density resolution obtained from specimens with diameters ranging from a few millimeters to centimeters is needed. Such a comparison should include the instrumental and analysis efforts as well as the potential benefit from a combination of approaches. Currently, especially X-ray grating interferometry (XGI) ${ }^{1-14}$ and propagation-based phase retrieval methods including holotomography $(\mathrm{HT})^{15-17}$ and single-distance phase tomography using Paganin's method $^{18,19}$ are successfully applied to generate threedimensional datasets of animal and human tissues with isotropic voxel sizes. ${ }^{20,21}$ The question arises: "Which performance is reached applying the methods mentioned above to soft tissues?" Here, we have chosen a tumor from cancerous cells grown in a Balb/c nude mouse and an entire heart of a male Fischer rat.

One of the difficulties in comparing phase contrast methods with each other is that they generally give different types of signals. This may be one of the reasons why there is very little literature on experimental comparisons so far.

An interesting approach was presented by Diemoz et al. who developed ${ }^{22}$ and applied ${ }^{23}$ a formalism for comparison that is based on specific image features in phase-contrast images. Sun et al. ${ }^{20}$ have reported a short qualitative comparison of PBI vs. XGI images. Here, we make a quantitative comparison based on an analysis of resolution, statistical properties, and fidelity of reconstructed values. This work is a continuation of an earlier paper. ${ }^{21}$ The present paper goes beyond this first study not only in terms of the depth of investigation but also in that the range of phase imaging methods compared is extended to include, notably, singledistance phase retrieval (SDPR).

\section{MATERIALS AND METHODS}

\section{A. Specimen preparation}

\section{Murine tumor}

The animal experiments with Balb/c nude mice (Charles River Laboratories, L'Arbresle, France), weighing between 22 and $25 \mathrm{~g}$, were performed at the Institute for Biomedical Engineering (ETH Zurich, Switzerland) in strict adherence to the Swiss law for animal protection. In order to obtain the murine tumors, a suspension of 1000000 cells that originated from the C51 colon cancer cell line was injected subcutaneously in the left flank of each mouse. The animals were monitored daily for health conditions and tumor growth. The tumor was measured by means of calliper or in vivo magnetic resonance imaging (MRI). Before the tumors reached a diameter of $3 \mathrm{~mm}$, usually 6-8 days after inoculation with tumor cells, mice were administered an anaesthesia and perfused with phosphate buffered saline solution (PBS) and para-formaldehyde 5\% (PFA) in order to fixate the tissues. Subsequently, the tumors were extracted and stored in 5\% PFA solution at a temperature of $4{ }^{\circ} \mathrm{C}$ until beamtime at a synchrotron radiation facility became available. A few days before the beamtime, the immersed tumors were put in a desiccator for a period of $12 \mathrm{~h}$ at a pressure of 5 millibars to reduce the frequently observed bubble formation during the high-resolution tomography experiment. After this procedure, the tumors were transferred into polymer pipette tips and stabilized with parts of plastic straws to prevent movement of the specimen within the pipette tip during rotation for data acquisition.

\section{Murine heart}

The heart specimen was obtained from a male Fischer rat (Charles River Laboratories, L'Arbresle, France) at the European Synchrotron Radiation Facility (ESRF, Grenoble, France). All operative procedures related to animal care of the rat were carried out strictly conformed to the Guidelines of the French Government. The animal did not show any pathological sign. After extraction, the specimen was immersed in $10 \%$ PFA solution. For the experiment, the heart was placed and stabilized in a plastic tube with a diameter of $11 \mathrm{~mm}$.

\section{B. Data acquisition and processing}

The imaging experiments were carried out at the beamline ID19 at the ESRF. The nominal source size was $135(\mathrm{~h}) \times 25(\mathrm{v}) \mu \mathrm{m}^{2}$, and the experimental station was located $150 \mathrm{~m}$ from the source. The technical details of the beamline at the time of the experiment are described elsewhere. ${ }^{24}$ An undulator source was used in combination with 
a multilayer monochromator $\left(\Delta \lambda / \lambda=10^{-2}\right)$ for the tumor measurement. A wiggler source and a double crystal monochromator $\left(\Delta \lambda / \lambda=10^{-4}\right)$ in Bragg geometry were used for the scans of the heart. The X-ray energy was $17.6 \mathrm{keV}$ and $19 \mathrm{keV}$, respectively, for the tumor and heart measurements. The photon flux at these experimental conditions was on the order of $10^{11}$ photons per second and per $\mathrm{mm}^{2}$.

For the tumor measurements, the detector setup consisted of a 19- $\mu$ m-thick europium-doped gadolinium gallium garnet scintillator, microscope optics with a $4 \times$ objective and a $2.5 \times$ eyepiece. The CCD detector was an ESRF FReLoN camera with $2048 \times 2048$ pixels of $15 \mu \mathrm{m}$ size. $^{25}$ The pixels were binned by a factor of $2 \times 2$, resulting in an effective grid of $1024 \times 1024$ pixels with a pixel size of $3.0 \mu \mathrm{m}$. For the measurements on the rat heart, a gadox (gadolinium oxysulfide) scintillator and $2 \times$ magnifying lens optics were used with two different detector heads: a FReLoN with $2048 \times 2048$ pixels of $15 \mu \mathrm{m}$ size for XGI and a FReLoN with $2048 \times 2048$ pixels of $14 \mu \mathrm{m}$ size for propagation-based imaging, resulting in respective effective pixel sizes of 8.0 and $7.5 \mu \mathrm{m}$.

\section{XGI}

Figure 1(a) schematically shows the setup of an X-ray grating interferometer, consisting of a phase grating $\mathrm{G}_{1}$ and an absorption grating $\mathrm{G}_{2}$. XGI yields differential phase contrast (DPC), i.e., the first derivative $\partial \varphi(x, y) / \partial x$ of the wavefront phase $\varphi$ along the direction $x$ perpendicular to the grating lines. ${ }^{11}$ The phase-stepping technique was used to recover the DPC and absorption signals. ${ }^{12}$ The description of the instrument installed at ID19 can be found in the literature. ${ }^{26}$

The interferometer used for the measurements on the tumor consisted of a $\pi$-shifting silicon phase grating $G_{1}$ and a

(a)

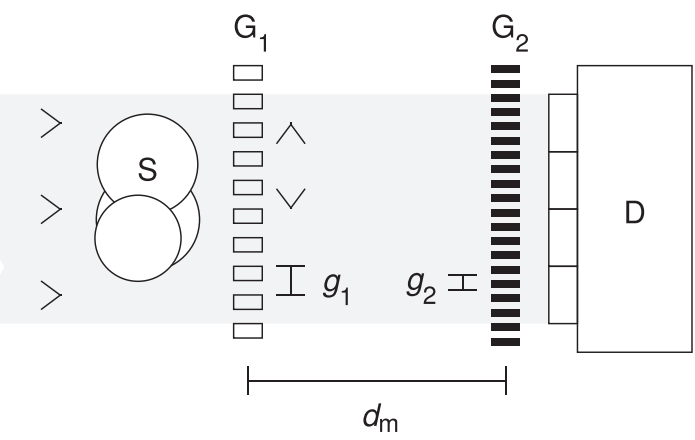

(b)

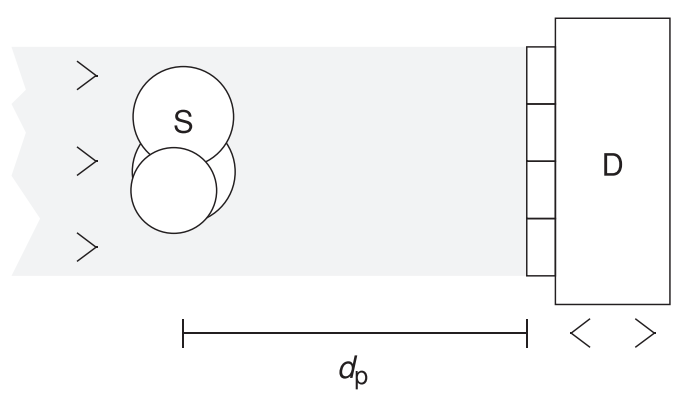

FIG. 1. Setup schemes for (a) grating interferometry and (b) propagationbased imaging. S: specimen; D: detector; $\mathrm{G}_{1}$ : phase grating; $\mathrm{G}_{2}$ : absorption grating. gold analyzer grating $\mathrm{G}_{2}$, placed at the fifth fractional Talbot distance (FTD) corresponding to $140 \mathrm{~mm} . \mathrm{G}_{1}$ had a period $p_{1}$ of $4.0 \mu \mathrm{m}$, while $\mathrm{G}_{2}$ had a period $p_{2}$ of $2.0 \mu \mathrm{m}$. The grating $\mathrm{G}_{1}$ was scanned over one fringe period in four steps. The exposure time was $0.3 \mathrm{~s}$ per raw detector image for 359 projection angle intervals over $360^{\circ}$. Because of the strong phase gradient between the edges of the sample container and the surrounding air, phase wrapping artifacts occurred. ${ }^{27}$ They were corrected, prior to tomographic reconstruction, using a priori information about the composition and geometry of the container.

The measurement on the heart specimen was also performed using a $G_{1}$ made of $\mathrm{Si}$ and a $\mathrm{G}_{2}$ made of $\mathrm{Au}$, but the grating periods, $p_{1}=4.8 \mu \mathrm{m}$ and $p_{2}=2.4 \mu \mathrm{m}$, were different from the tumor measurements. The intergrating distance was $482 \mathrm{~mm}$, corresponding to the eleventh FTD. Grating $\mathrm{G}_{1}$ was scanned over one fringe period in five steps, with an exposure time of $1.5 \mathrm{~s}$ per raw image. This phase-stepping scan was repeated for 1499 angular intervals over $360^{\circ}$. A software correction of phase wrapping effects was not necessary in this case because the heart in its container was immersed in a tank filled with water, so that the phase gradient at the container walls was significantly reduced.

\section{2. $H T$}

HT is based on phase contrast generated by the propagation of $\mathrm{X}$ rays in free space between specimen and detector. ${ }^{15}$ The additional information with respect to absorption imaging to solve the phase problem is obtained by taking images for at least two propagation distances. In the present study, the propagation distances were chosen by optimizing the overall squared contrast transfer function $(\mathrm{CTF})^{28}$ while taking into account additional constraints, such as technical limitations of propagation distance.

The HT data on the tumor specimen were measured at four distances: 13, 60, 120, and $360 \mathrm{~mm}$. For each of these distances, images were taken at 800 equidistant angles over a range of $180^{\circ}$, with an exposure time of $0.3 \mathrm{~s}$ per raw image. Alignment of the dataset acquired at $13 \mathrm{~mm}$ with the other images was impossible due to the poor signal in the image taken at the shortest propagation distance, and the holotomographic phase retrieval, and, consequently, the tomographic reconstruction, was performed excluding this dataset.

For the heart measurements, data were taken at five propagation distances: 200, 400, 700, 900 and $1100 \mathrm{~mm}$. The exposure time was $1 \mathrm{~s}$ per raw image for 1499 angular steps over $360^{\circ}$. In these measurements, the accessible sampledetector distances were technically limited to $1.1 \mathrm{~m} .^{24}$ The hardware alignment at $900 \mathrm{~mm}$ of the detector with the $\mathrm{X}$-ray beam failed in this measurement (the sample was not entirely in the field of view of the detector), and the dataset at this distance was not used for the holotomographic reconstruction.

The holotomographic phase retrieval software at beamline ID19 gives users the choice between several algorithms. For the present study, a mixed approach between the CTF and the transport of intensity equation (TIE) was chosen. ${ }^{16,29,30}$ This combined model is valid for extended 
propagation distances as well as non-negligible absorption. To reduce low-frequency artifacts, which can arise from the fact that the transfer function for phase contrast is small in the low spatial frequency range, the low-frequency components of the phase profile are presumed to be proportional to the logarithm of the projected intensity transmission via a fixed estimated ratio $\delta / \beta$ of refractive index decrement over absorption coefficient (although more recent work extends this approach to inhomogeneous objects $\left.{ }^{31}\right) .{ }^{16}$ In the present case, this a priori input parameter was taken to be 1371 for the tumor and 1546 for the heart, corresponding to the values of $\delta$ and $\beta$ of water at 17.6 and $19 \mathrm{keV}$.

To improve the phase retrieval, the HT reconstruction procedure includes, by default, a deconvolution of the projection data with an experimentally determined detector point spread function. This deconvolution has been applied to both tumor and heart data. Moreover, to study the effects of the deconvolution on the quality of the volumes, HT reconstructions of the heart specimen without deconvolution has also been performed. Note that neither the singledistance phase retrieval software nor the software to process the XGI data correct for the detector point spread function.

\section{SDPR}

Under simplifying assumptions, the phase profile $\varphi(x, y)$ of the X-ray wavefront at the exit plane of the specimen can be recovered from only a single radiograph in free-space propagation phase contrast, taken at a suitable distance between detector and specimen. ${ }^{18,19,32}$

Paganin's algorithm assumes that the ratio $\delta / \beta$ of the decrement of refractive index $\delta$ over the absorption coefficient $\beta$ should be constant throughout the specimen. While this implicitly allows for non-negligible absorption by the specimen, it also means that the composition of the specimen should vary only marginally within the object. It should be noted that, while the multi-distance HT approach used in this study (Sec. II B 2) also assumes such a fixed ratio $\delta / \beta$, it only uses this fixed ratio for the reconstruction of the low spatial frequencies, and only when necessary. In Paganin's algorithm, however, the assumption of such a fixed ratio should be expected to have much greater influence since it affects the whole frequency spectrum of the retrieved phase map.

Moreover, Paganin's algorithm has been developed from the transport-of-intensity equation and implies that propagation distances are limited to $d_{\mathrm{p}} \ll D^{2} / \lambda$, where $D$ is the size of the smallest feature to be resolved in the object. With the assumption that $D$ is three pixels large, for the Paganin's algorithm to be valid, $d_{\mathrm{p}}$ should be less than $1.1 \mathrm{~m}$ for the tumor data and less than $8 \mathrm{~m}$ for the heart data. This condition is satisfied in our measurements.

In this work, we have used the freely available program ANKAphase, ${ }^{33}$ which implements Paganin's algorithm. Phase recovery using ANKAphase was performed for all data sets obtained by free-space propagation, in addition to the HT reconstruction performed on the same data. The assumed ratios $\delta / \beta$ were the same as for the HT reconstruction.

\section{Relative dose}

The dose deposited in each sample was estimated to be on the order of MGy per scan. However, it was not the same for the measurements (relative doses are listed in Table I). For the tumor sample, the doses remain within the same order of magnitude for all methods, and within a factor of 2 between XGI and SDPR. For the heart specimen, however, the dose deposited for the XGI measurements is $7.5 \times$ as large as that deposited for the SDPR measurements (the HT measurements lying in between the two). The use of advanced acquisition methods for XGI, ${ }^{34}$ which were not applied here, could reduce dose without noticeable loss in image quality.

\section{Tomographic reconstruction and 3D registration}

After phase recovery, the tomographic reconstruction of the data was carried out using the program PyHST. ${ }^{35}$ It uses the filtered back-projection (FBP) method with a standard ramp filter, i.e., the Ram-Lak filter. Some of the data were tomographically reconstructed with code written in IDL, following the same principles (i.e., FBP and no high-frequency damping). For the DPC projection images from XGI data, an imaginary sign filter, ${ }^{36}$ implemented in PyHST, was used.

TABLE I. Results for the specimens investigated in this study. CNR: contrast-to-noise ratio; SR: spatial resolution; XGI: grating interferometry; SDPR: single distance phase retrieval using Paganin's algorithm; HT: holotomography. The dose values are relative to other measurements for the same sample. They do not permit a comparison between the data of different samples. The absorption values from PBI data have been calculated using the tomogram acquired at the shortest propagation distance with the weakest edge-enhancement signal.

\begin{tabular}{|c|c|c|c|c|c|c|c|c|c|c|c|c|c|}
\hline \multirow[b]{2}{*}{ Method } & \multicolumn{6}{|c|}{ Tumor } & \multicolumn{7}{|c|}{ Heart } \\
\hline & $\begin{array}{c}d_{\mathrm{p}} \\
(\mathrm{mm})\end{array}$ & $\begin{array}{c}\text { CNR } \\
\text { (II) }\end{array}$ & $\begin{array}{c}\text { CNR } \\
\text { (III) }\end{array}$ & $\begin{array}{l}\text { CNR } \\
\text { (IV) }\end{array}$ & $\begin{array}{c}\mathrm{SR} \\
(\mu \mathrm{m})\end{array}$ & $\begin{array}{l}\text { Rel. } \\
\text { dose }\end{array}$ & $\begin{array}{c}d_{\mathrm{p}} \\
(\mathrm{mm})\end{array}$ & $\begin{array}{c}\text { CNR } \\
\text { (II) }\end{array}$ & $\begin{array}{c}\text { CNR } \\
\text { (III) }\end{array}$ & $\begin{array}{l}\text { CNR } \\
\text { (IV) }\end{array}$ & $\begin{array}{c}\text { CNR } \\
(\mathrm{V})\end{array}$ & $\begin{array}{c}\text { SR } \\
(\mu \mathrm{m})\end{array}$ & $\begin{array}{l}\text { Rel. } \\
\text { dose }\end{array}$ \\
\hline XGI & $\ldots$ & 59.4 & 54.4 & 167.8 & 25 & 1.8 & $\ldots$ & 54.7 & 103.8 & 262.8 & 250.9 & 82 & 7.5 \\
\hline SDPR & 13 & 6.1 & 11.9 & 139.8 & 12 & 1.0 & 200 & 13.8 & 29.2 & 89.4 & 594.9 & 49 & 1.0 \\
\hline SDPR & 60 & 17.3 & 17.7 & 171.9 & 10 & & 400 & 13.6 & 35.6 & 103.7 & 879.6 & 40 & \\
\hline SDPR & 120 & 20.4 & 17.3 & 147.6 & 10 & & 700 & 24.1 & 82.6 & 256.1 & 539.9 & 39 & \\
\hline SDPR & 360 & 23.8 & 19.9 & 58.9 & 11 & & 1100 & 24.9 & 87.4 & 134.5 & 267.6 & 39 & \\
\hline $\mathrm{HT}$ & $\ldots$ & 11.1 & 11.7 & 97.3 & 8 & 3.0 & $\ldots$ & 9.3 & 97.9 & 112.8 & 428.0 & 40 & 4.0 \\
\hline HT without det. deconv. & $\ldots$ & $\ldots$ & $\ldots$ & $\ldots$ & $\ldots$ & & $\ldots$ & 15.9 & 84.1 & 114.6 & 319.4 & 70 & \\
\hline Absorption from PBI data & 135 & 0.1 & 0.4 & 8.8 & 6 & 1.0 & 200 & 0.7 & 20.1 & 3.5 & 32.3 & 37 & 1.0 \\
\hline Absorption from XGI data & $\ldots$ & 1.5 & 12.2 & 5.2 & 19 & 1.8 & $\ldots$ & 0.6 & 13.3 & 9.5 & 56.5 & 59 & 7.5 \\
\hline
\end{tabular}


This corresponds to the ramp filter used for the other datasets, with the difference that it incorporates the integration step necessary for correct phase retrieval.

Since all of the phase contrast methods used here are differential, an integration constant was added to each tomogram, to obtain absolute values of the decrement $\delta$ of refractive index. This constant was obtained by shifting the histogram of the tomogram data so that the peak corresponding to the environment surrounding the container corresponded to the literature value of $\delta$ for this specific material. For the PBI measurements, this material was air, with a value of $\delta_{\text {air }}=7 \times 10^{-10}$. For the XGI measurements of the heart, the material was demineralized water at room temperature. The literature value for $19 \mathrm{keV}$ is $\delta_{\text {water }}=6.39 \times 10^{-7.37}$

For a detailed comparison between the methods for each specimen, equivalent regions of interest (ROIs) had to be identified. For the measurements of the heart, this was trivial because the object had not been removed from its container between the data acquisition of XGI and HT. The tumor sample, however, had been removed from its container between the two measurements to remove air bubble formed during the first HT tomography scan. The sample could not be placed in exactly the same position for the XGI scan and was therefore necessary to register the tomography volume images obtained by PBI with those from XGI. Using a 3D registration tool, ${ }^{38,39}$ the calculated transformation parameters were used to transform the XGI tomograms to match the ROI defined in the PBI data.

\section{RESULTS}

The tomographic slices upon which all further analysis was based are represented in Figs. 2-5. These figures show, for each imaging modality and phase-retrieval technique, one selected slice of the tumor and the heart. The positions of the slices in the entire volume data sets were selected for their wealth of features. The images are cropped around the sample region (i.e., the region occupied by the background material-water or air-was cropped out) for better visualization of the sample details.

Figs. 2 and 3 display images from the tumor data. The absorption tomogram (Fig. 2(a)) and the phase tomogram (Fig. 2(b)) of the XGI data were extracted from the same data. Figs. 2(c), 2(d), and 3 show images obtained from free space propagation data. Figure 2(c) is an absorption tomography slice (i.e., no phase recovery was performed), reconstructed from data taken at the smallest propagation distance realized $\left(d_{\mathrm{p}}=13 \mathrm{~mm}\right)$. Figure 2(d) shows a phase tomography slice obtained by HT. Figure 3 shows phase tomograms obtained with SDPR to the data sets taken at different distances.

Figs. 4 and 5 show data for the heart, arranged as the tumor data in Figs. 2 and 3, respectively.

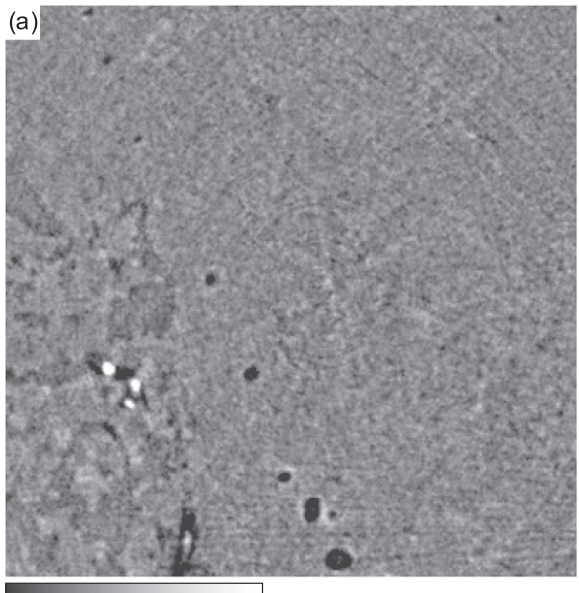

$\begin{array}{llll}0 & 2 & 4 & \mu(1 / \mathrm{cm})\end{array}$

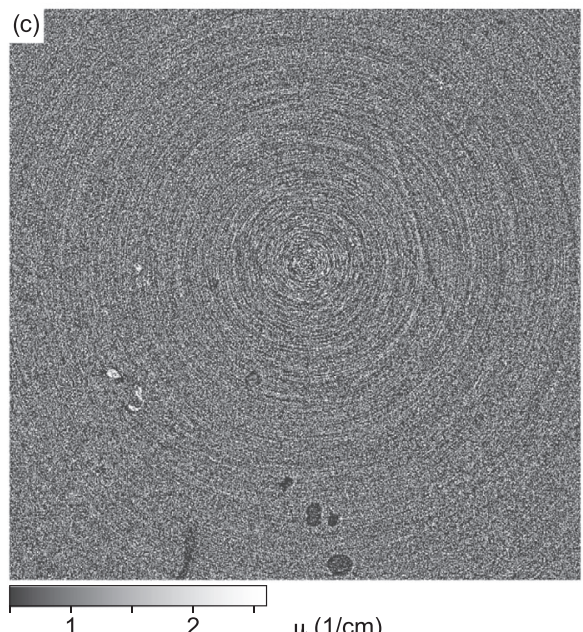

$\begin{array}{lll}1 & 2 & \mu(1 / \mathrm{cm})\end{array}$

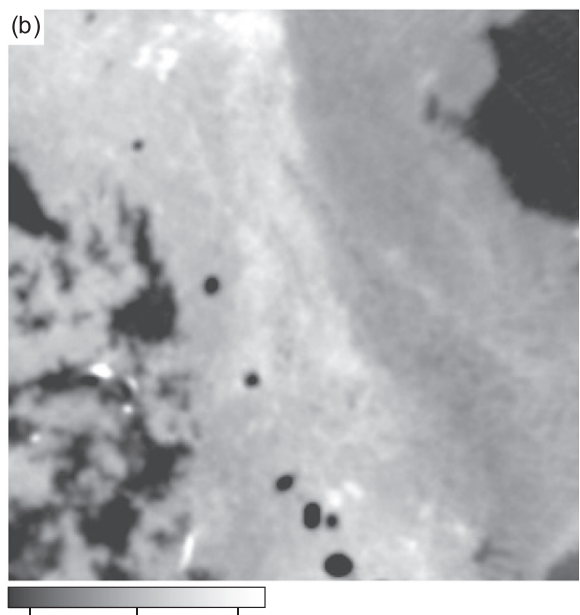

7.1

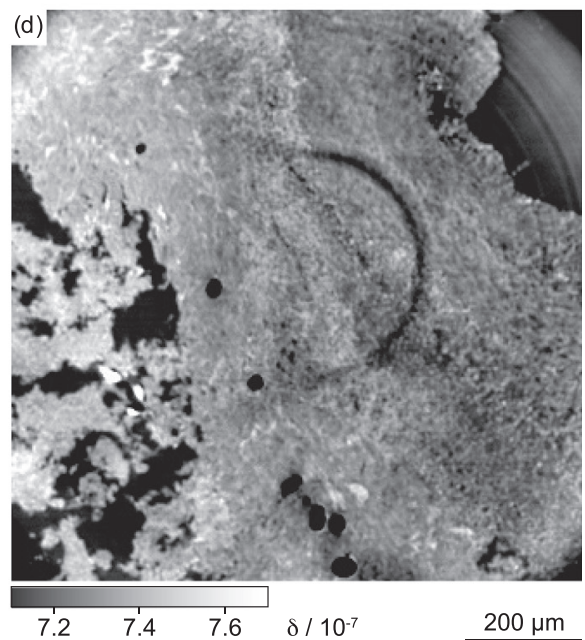

FIG. 2. Tomographic slices of the tumor sample. (a) Absorption tomogram from XGI data, (b) phase tomogram from XGI data, (c) absorption tomogram reconstructed from data measured at the distance $d_{\mathrm{p}}=13 \mathrm{~cm}$, and (d) phase tomogram from HT data. 

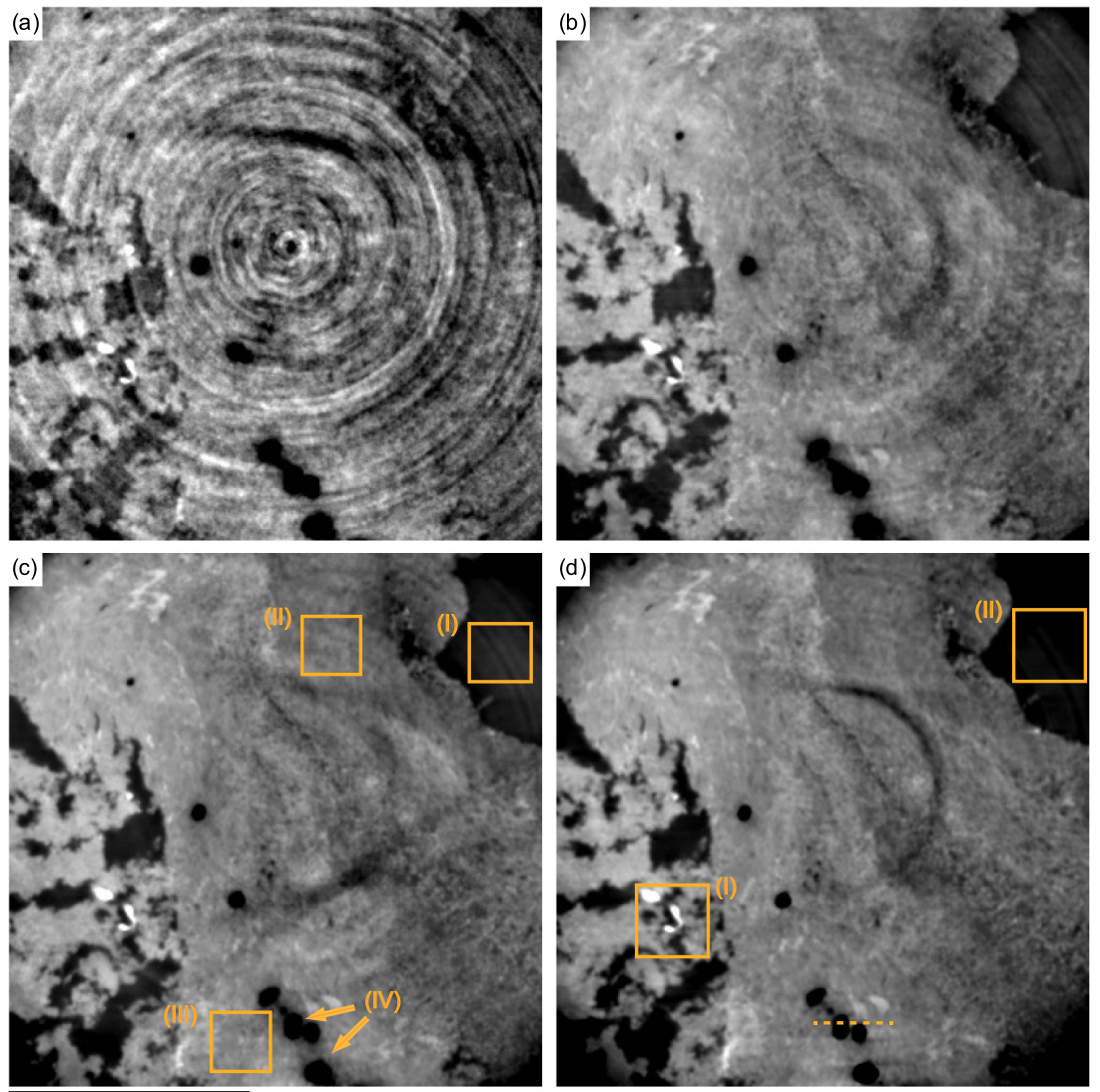

$\begin{array}{llll}7.8 & 8.0 & 8.2 & \delta / 10^{-7}\end{array}$
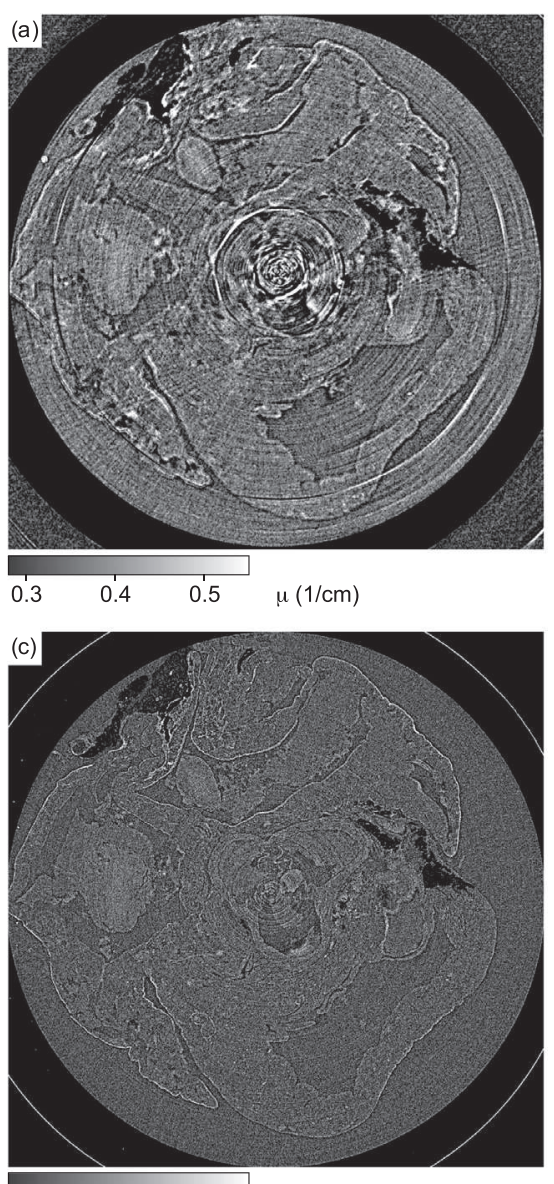
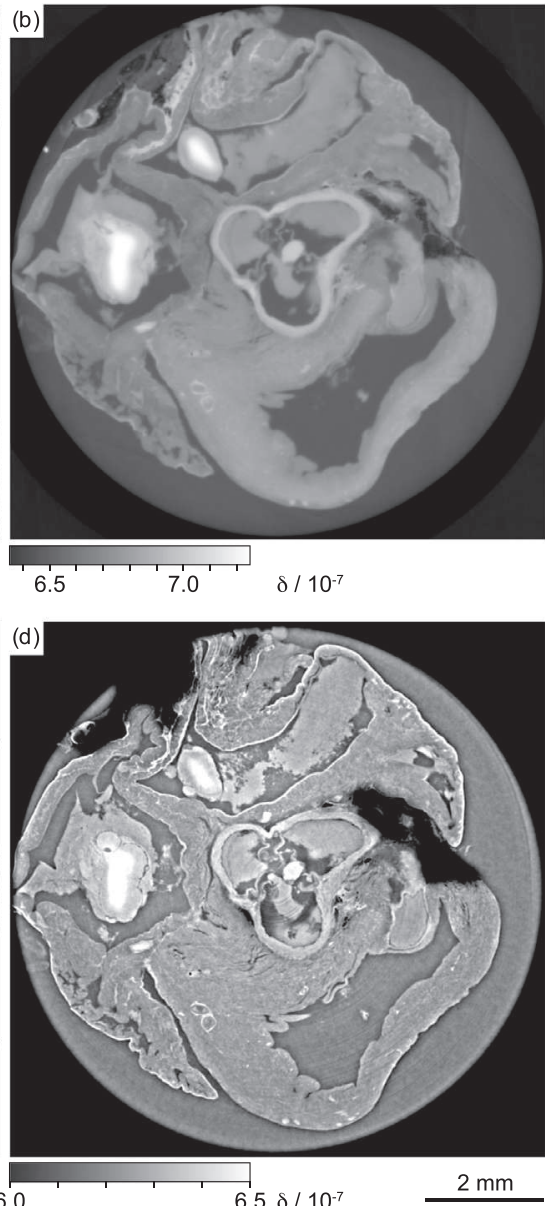

FIG. 3. Tomographic slices of the tumor obtained with SDPR. The images in panels (a) to (d) correspond to propagation distances of $13,120,180$, and $360 \mathrm{~mm}$. The regions of interest indicated in panel (c) are those that were used for the calculation of the CNR: PFA (I), dark tissue (II), bright tissue (III), and lipid (IV). The rectangles in panel (d) show the regions for which the RSP was determined: features (I) and background (II). The dashed line indicates the position at which the profiles shown in Fig. 7(a) were taken.
FIG. 4. Tomographic slices of the rat heart. (a) Absorption tomogram from XGI data, (b) phase tomogram from XGI data, (c) absorption tomogram reconstructed from data measured at the distance $d_{\mathrm{p}}=200 \mathrm{~cm}$, and (d) phase tomogram from HT data. 

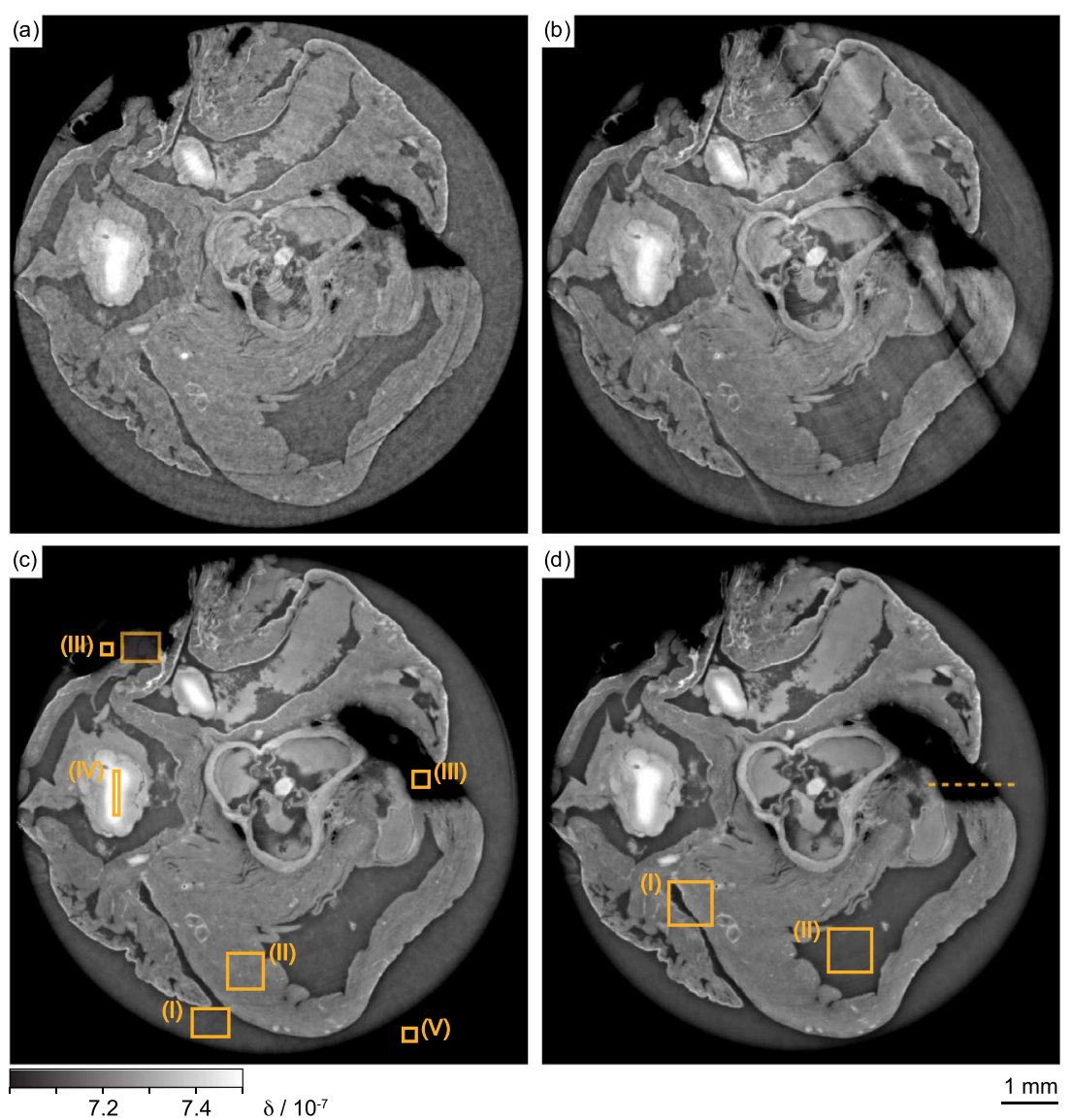

FIG. 5. Tomographic slices of the rat heart obtained with SDPR. The images in panels (a) to (d) correspond to propagation distances of 200, 400, 700, and $1100 \mathrm{~mm}$. The regions of interest indicated in panel (c) are those that were used for the calculation of the CNR: formalin (I), tissue (II), lipid (III), and blood (IV). The rectangles in panel (d) show the regions for which the RSP was determined: features (I) and background (II). The dashed line indicates the position at which the profiles shown in Fig. 7(b) were taken.
As expected, these specimens yield hardly any absorption contrast for the photon energies applied. This is apparent in the absorption contrast tomograms (Figs. 2(a), 2(c), 4(a), and 4(c)). Most of the few details visible in these images are actually a result of edge-enhancing inline phase contrast, which could be revealed because of the high coherence of the $\mathrm{X}$-ray beam and the relatively large propagation distance with respect to the pixel size.

The phase images, on the other hand, highlight fine details of the anatomy of the tumor and the heart specimens. Among them, we note that in the XGI image of the tumor (Fig. 2(b)), two types of soft tissue can be distinguished (denser at the left and darker at the right of the image). In phase images of the heart, the tricuspid aortic valve is clearly visible at the center. In both samples, the low-density spots (appearing in black) are assumed to be lipid cells, while blood vessels can be seen as bright disordered spots, indicating their higher density with respect to the surroundings. The dark background is the PFA.

To compare the quality of the images obtained by the different modalities and reconstruction algorithms, we consider the spatial resolution and the density resolution as well as the presence of artifacts.

The $\delta$ resolution, has been calculated by computing the contrast-to-noise ratio (CNR), which is defined as ${ }^{40}$

$$
\mathrm{CNR}=\frac{\left|S_{a}-S_{b}\right|}{\sqrt{\sigma_{a}^{2}+\sigma_{b}^{2}}},
$$

where $S_{a}$ and $S_{b}$ are the mean values of two homogeneous ROIs $a$ and $b$ in the tomograms, representing two different materials. The quantities $\sigma_{a}$ and $\sigma_{b}$ denote the standard deviations of the values in these two ROIs of identical size. The CNR was determined for different pairs of materials $a$ and $b$, where $b$ was always the PFA. The ROIs used for the CNR calculation are indicated with rectangles in Figs. 3(c) and 5(c).

As a measure of the spatial resolution in the tomograms, the radial spectral power function (RSP) was calculated for regions with high-contrast features and normalized by the RSP of a homogeneous area. These regions are marked in Figs. 3(d) and 5(d). The spatial resolution was estimated as the highest spatial frequency for which the RSP is greater than twice the baseline value. ${ }^{41,42}$

The values of CNR and spatial resolution calculated in this way are listed in Table I.

In addition to the CNR, the histograms of the presented tomographic slices were calculated. The histogram analysis gives additional information on the density resolution of the imaging modalities as the width of the peak can be considered as a measure of the density resolution: the narrower the peak, the higher the density resolution. ${ }^{43}$

The histograms of the entire tomograms are shown in Fig. 6. In addition, section profiles were taken at the positions marked by the dashed lines in Figs. 3(d) and 5(d). These profiles are plotted in Fig. 7.

The study was completed by a qualitative visual inspection and comparison of the tomograms obtained with the imaging methods, including such aspects as the distinction of different regions and the presence of noise and artifacts, such as rings, cupping or other low-frequency artifacts. 
(a) Tumor

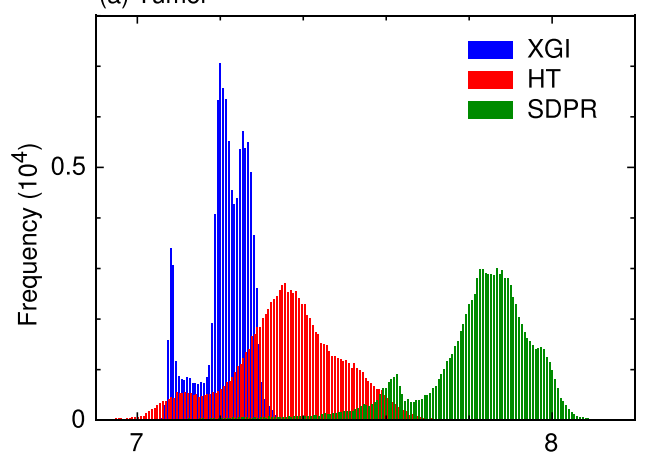

Real part of the refractive index $\delta\left(10^{-7}\right)$

(b) Heart
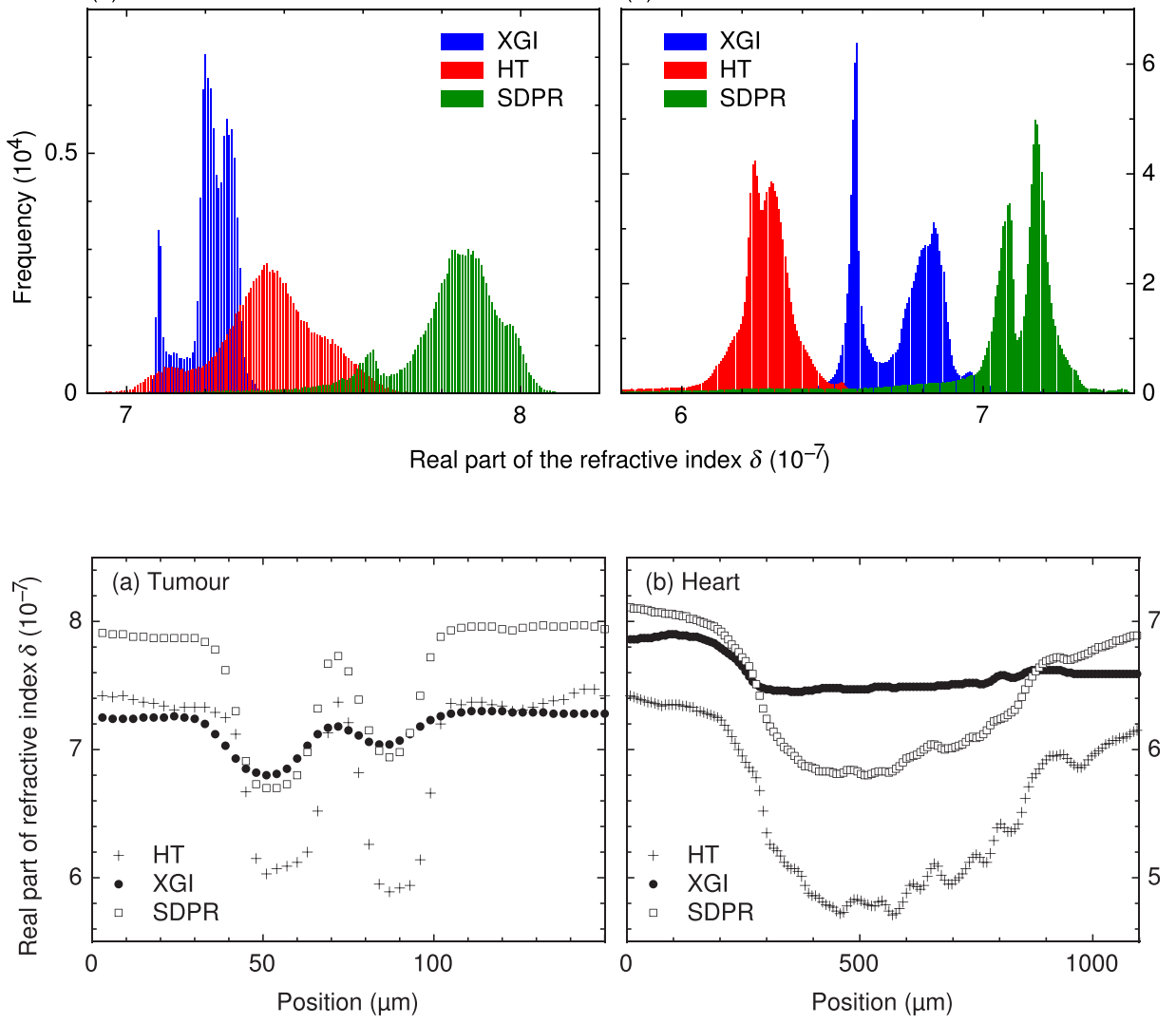

FIG. 6. Histograms of the phase slices of the tumor (a) and heart (b) obtained with the different methods. For the SDPR method, the histogram of the slice obtained at the largest distance is plotted here: $360 \mathrm{~mm}$ for the tumor and $1100 \mathrm{~mm}$ for the heart.
The phase retrieval algorithm for holotomography data contains a filter that performs a deconvolution of the image with the resolution-function of the detector. To obtain better insight in the influence of this filter on the image quality, the holotomography data of the heart were processed additionally without the filter. A comparison of the heart data obtained with and without the deconvolution is presented in Fig. 8.

\section{DISCUSSION}

The nearly complete absence of absorption contrast is reflected by the poor CNR values of the absorption images (Table I). Visual inspection reveals that the absorption images are dominated by noise and other artifacts, such as
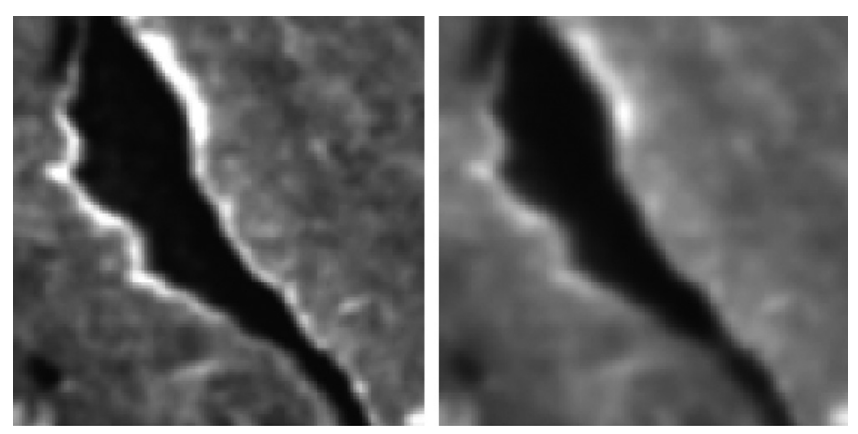

FIG. 8. Details of tomographic slices of the heart obtained with HT, with (left) and without (right) deconvolution with the detector point-spread function. ring artifacts, and cannot be used for practical purposes, reflecting the fact that absorption contrast is insufficient for the discrimination of soft-tissue components.

Unlike the absorption data, most of the phase tomograms are of high quality in terms of their contrast-to-noise properties. This observation is valid for images obtained from the three phase retrieval methods investigated, i.e., XGI, HT, and SDPR. Nonetheless, substantial differences exist between the images obtained with the methods, and/or from relative propagation distances. They are discussed below.

The overall best results in terms of the CNR are obtained by XGI. For most of the materials in the specimens, the CNR values shown in Table I are the highest for the XGI data. This is particularly true for tissue and blood. The trend is different for lipid, where the CNR values are similar for PBI and XGI, and for the container material, where PBI yields much higher CNR values.

The lipid and the container material (polypropylene, $\mathrm{PP})$ are structures yielding relatively strong phase contrast. Closer inspection of the data reveals that the higher CNR for these materials obtained with the PBI techniques compared to XGI is mainly due to the fact that the contrast at which these structures are rendered is larger in the phaseretrieved PBI images than in XGI; i.e., the numerator in Eq. (3) takes a higher value. For example, the section profiles in Fig. 7 demonstrate that the reconstructed refractive index of the lipid structures in the tumor differs from the surrounding tissue by no more than $3 \times 10^{-8}$ in the XGI tomogram, whereas the difference exceeds $10^{-7}$ in the PBI images (both SDPR and HT). The reconstructed values of $\delta$ for the 
container material are even extremely different between XGI and the PBI methods: XGI yields values around $6 \times 10^{-8}$ (close to the literature value of PP for the photon energies used), while HT and SDPR yield underestimated values around $3 \times 10^{-8}$.

These discrepancies in the reconstructed values of $\delta$ can have various origins. ${ }^{31}$ In the PBI techniques, the condition of a known ratio $\delta / \beta$ may be violated to an extent that can explain the differences. This is clearly the case for the container material, for which $\delta / \beta$ ranges between 5000 and 6500 for the photon energies used, i.e., more than 3 times higher than the values for water, which were assumed to be uniformly valid in the slice. XGI does not depend on such a priori assumptions and yields more reliable values for these high-contrast materials. However, it is known that XGI may yield systematically reduced contrast if phase wrapping occurs. ${ }^{27}$

Visual inspection of Figs. 3 and 5 shows that the images obtained by SDPR in general improve in phase contrast as the propagation distance $d_{\mathrm{p}}$ is increased. This is because phase contrast increases with distance, and, consequently, also the contrast in the phase tomogram becomes higher for larger sample-to-detector distances. This effect is confirmed by the CNR values extracted from the SDPR images (Table I) which increase, for almost all the materials, with the propagation distance. However, the CNR values obtained for lipid in the tumor and for the container of the heart, which are details providing strong contrast already at short distances, do not follow this trend. Figs. 3 and 5 also show that image artifacts, such as ring artifacts, are less pronounced in the images taken at large $d_{\mathrm{p}}$. This can be attributed to the fact that, while the phase contrast in general increases with distance, the prevalence of artifacts, which arise predominantly from imperfect correction of sharply localized variations in the beam profile or non-uniformity of the detector response (e.g., impurities on the scintillator), is essentially independent of distance.

It is interesting to notice that, in our measurements, the HT images show CNR values which are comparable or slightly inferior to those obtained from the single-distance data.

The use of the detector deconvolution filter in HT drastically improves the spatial resolution (Table I). In terms of the statistical properties of the images, one should expect the CNR to decrease as a result of the use of this filter (which acts like a sharpening filter and can thus be expected to amplify noise). However, this is not generally the case, as a comparison of the CNR values obtained with and without the filter shows (Table I). The fact that regions as the lipid or the container wall exhibit a better CNR with the filter activated than without it may be due to the fact that the sharpened projection images reduce cupping artifacts in the tomograms. We note that the same filter can, in principle, be combined with the other phase retrieval methods, although this was not implemented in the software used for this study.

The spatial resolution, as assessed qualitatively through inspection of feature edges in the images and estimated quantitatively by the RSP method (Table I), is clearly better in PBI than in XGI. This finding concerns both the absorption images and the phase tomograms. We believe that the main reason for this systematic difference between PBI and XGI is that, while the resolution is limited by X-ray diffraction in both cases, the mechanism is different. In XGI, the spatial resolution is influenced by the shear between the two interfering first orders of diffraction of the grating $G_{1}$. This shear is

$$
s=2 \frac{\lambda d_{m}}{p_{1}}
$$

Applied to the experimental parameters, Eq. (4) yields shear values of $s=9.9 \mu \mathrm{m}$ for the tumor measurements and $s=26.2 \mu \mathrm{m}$ for the heart measurements.

In our propagation-based images, the spatial resolution is mainly affected by the width $w$ of the first Fresnel zone

$$
w=\sqrt{\lambda d_{\mathrm{p}}}
$$

The values of $w$ for the PBI measurements on the tumor are up to $w=5.0 \mu \mathrm{m}$ for $d_{\mathrm{p}}=360 \mathrm{~mm}$, and for the rat heart, they are up to $w=8.5 \mu \mathrm{m}$ for $d_{\mathrm{p}}=1100 \mathrm{~mm}$. The observation of poorer spatial resolution in XGI compared to PBI is thus in qualitative accordance with the expectations.

The HT images (Figs. 2 and 4) clearly have the best spatial resolution among the tomograms obtained in this study, for each of the specimens. This is confirmed by the RSP analysis (Table I) and by a look at zoomed details (Fig. 9). This result is expected because (i) HT combines the high contrast of long propagation distances with the highresolution information that can be obtained at short distances, and (ii) among the different computer programs used in this study for retrieving the phase images, the HT routine was the only one with a detector deconvolution routine. The fact that the HT reconstruction of the heart data set without the deconvolution yields a spatial resolution value that is poorer even than the reconstructions obtained without such a filter using SDPR indicates that the deconvolution filter is decisive for spatial resolution.

In the case of the heart, one of the visual differences between the XGI tomograms on the one hand and the PBI images on the other is that the PBI data exhibit remaining phase-contrast fringes even after phase retrieval (Figs. 4(d) and 5), while the XGI data do not exhibit any such artifact (Fig. 4(b)). These residual fringes in the PBI images can have a substantial influence on any quantitative analysis of phase tomography data. In the present case, they may have affected the results of resolution estimations by the RSP method, in which they tend to give deceivingly good values for spatial resolution. On the other hand, if such fringes are present in regions used for contrast analysis, they will degrade the CNR value and broaden the peaks in the histograms. For the CNR analysis, the analyzed regions were chosen to exclude these effects, but the histograms in Fig. 6 include them. More importantly, residual fringes will generally impair the correct interpretation of the reconstructed volume images, whether they are inspected by a human beholder or whether machine-aided segmentation procedures 

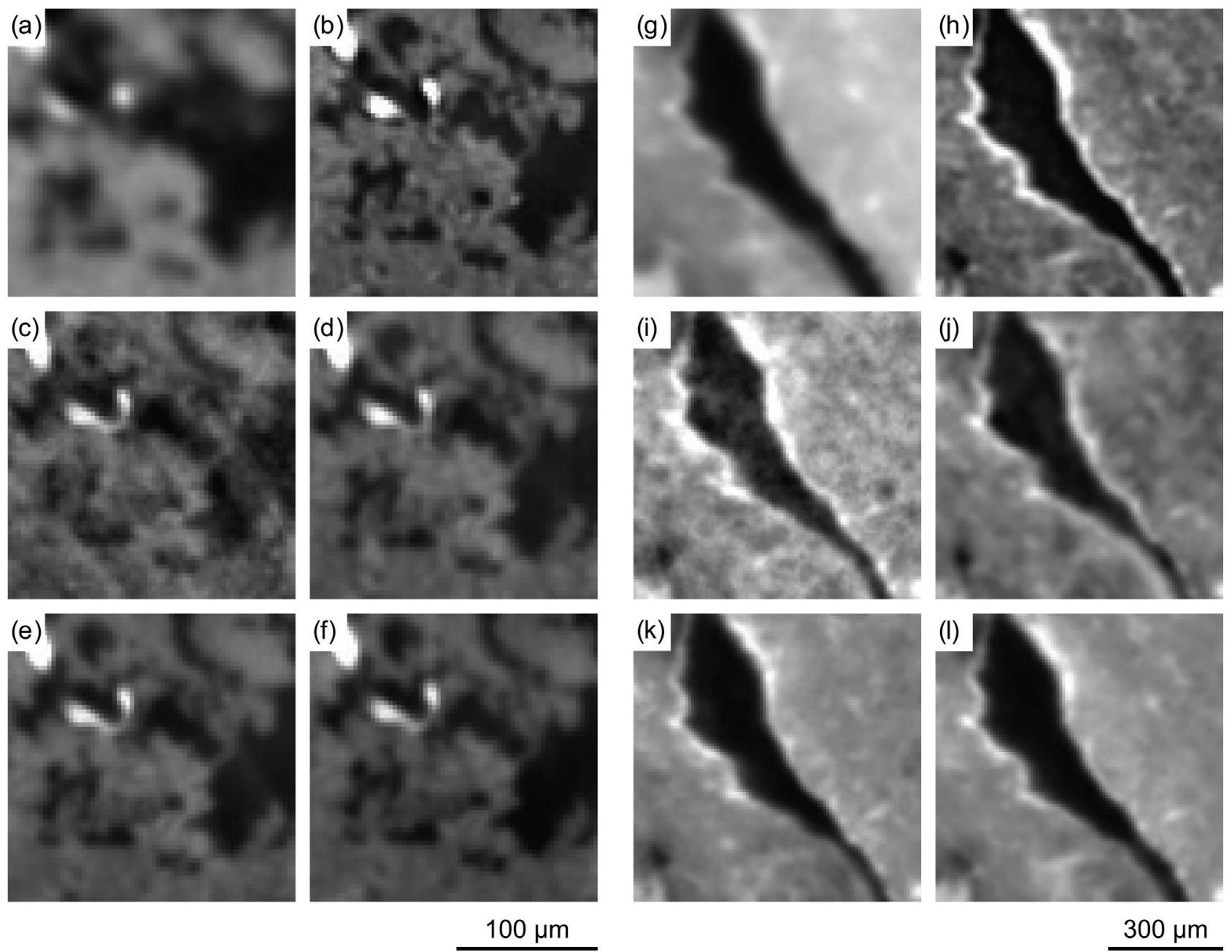

FIG. 9. Left: regions of interest of the phase tomograms of tumor from which the SR was estimated with the RSP method. The details correspond to images obtained with XGI (a), HT (b), SDPR with $d_{\mathrm{p}}=13,120,180$, and $360 \mathrm{~mm}$ (c)-(f). Right: regions of interest of phase tomograms of the heart from which the SR was estimated with the RSP method. The details correspond to images obtained with XGI (g), HT (h), SDPR with $d_{\mathrm{p}}=200,400,700$, and 1100 mm (i)-(1).

are used. In both cases, the artifacts pose the risk of falsepositive recognition of features such as interfacial layers.

\section{CONCLUSIONS}

The comparison of synchrotron-radiation X-ray phase tomography data obtained with different acquisition and phase-retrieval methods, as presented in this study, quantitatively confirms the complementarity of the three methods investigated, i.e., XGI and two phase recovery methods in PBI: multiple-distance HT in a combined TIE/CTF approach, and single-distance phase retrieval using Paganin's method, see Table II.

The XGI phase tomographic data have the highest density resolution and show the best overall accuracy of the retrieved values of refractive index (where those values were known). They do not show substantial problems with lowfrequency artifacts such as cupping and have no remaining phase-contrast fringes. This makes XGI phase tomograms easy to interpret and to segment. On the other hand, the spatial resolution of XGI is limited by the diffraction in the first grating. Moreover, phase-wrapping artifacts might occur when materials with strong refractive index differences are present in the sample. This type of artifacts might deteriorate quantitativeness in the reconstructed data and decrease spatial resolution. ${ }^{27}$

The HT approach used here performed best concerning spatial resolution. An important factor in this performance was the deconvolution with the estimated detector resolution carried out by the HT reconstruction software. Such a deconvolution filter would be a valuable option also in phase retrieval software for the other techniques.

SDPR has the advantage over XGI and HT that, apart from the possibility to place the detector at a suitable distance from the sample, it requires no more instrumental

TABLE II. Summary of the results of the experimental comparison study of three phase tomography methods. $\star \star \star$ : very good, $\star \star$ : good, $\star$ : needs improvement.

\begin{tabular}{lccc}
\hline \hline & XGI & SDPR & HT \\
\hline spatial resolution & $\star \star$ & $\star \star \star$ & $\star \star \star$ \\
density resolution & $\star \star \star$ & $\star \star$ & $\star \star$ \\
quantitativeness & $\star \star \star$ & $\star$ & $\star$ \\
data acquisition speed & $\star$ & $\star \star \star$ & $\star \star$ \\
simplicity of data processing & $\star \star$ & $\star \star \star$ & $\star$ \\
simplicity of setup & $\star$ & $\star \star \star$ & $\star \star$ \\
\hline \hline
\end{tabular}


effort than a standard absorption tomography scan, both in terms of the experimental procedure (in particular the net exposure time and the total time required for data acquisition) and the amount of raw data generated. Moreover, because SDPR relies in the acquisition of only one projection per viewing angle, it is the only phase-contrast method, among those studied here, that can be used for time-resolved studies. However, reconstructions using SDPR requires a reasonable estimate of $\delta / \beta$, and has the important restriction that acceptable results are only obtained for objects in which the actual ratio $\delta / \beta$ does not deviate significantly from the assumed value.

From a user's point of view, it can be noted that there is generally a tradeoff between complexity of the setup, complexity of the data processing, and quality of the data. Among the methods used here, XGI is the one that requires the most complex mechanical setup. However, the data analysis requires no other parameters than the experimental geometry, and, in particular, no knowledge of the sample composition. The processing is thus, in principle, fully automatic. An important restriction is, however, the need to avoid phase wrappings. The sample therefore either needs to be immersed in a phase-matching liquid (here: water) or the outer edges of the sample (or its container) need to be replaced in the projection radiographs by modeled data, an approach chosen for one of the samples measured in this study.

Holotomography, on the other hand, is conceptually simple in terms of data acquisition. The difficulty lies in the processing, which requires substantial manual interaction and a relatively high degree of expertise. It also requires image registration of the data taken at different distances. While this step is largely automated, it may fail or yield unsatisfactory results, especially for samples that exhibit weak contrast.

\section{ACKNOWLEDGMENTS}

The authors gratefully acknowledge Jean-Paul Valade (ESRF) for his help in developing and building the mechanics of the high-resolution interferometer, and Alessandro Mirone (ESRF) for implementing the imaginary sign filter into the PyHST software. Elena Reznikova, Johannes Kenntner, Thomas Grund, and Martin Börner (KIT) and Christian Grünzweig, Jens Bruder, and Simon Rutishauser (PSI) made the gratings used in the interferometers. Hans Deyhle (U Basel) provided assistance during data acquisition and treatment. The project was supported by the Swiss National Science Foundation (Grant No. 200021_127297/1) and by the Karlsruhe Nano Micro Facility, a Helmholtz Research Infrastructure at Karlsruhe Institute of Technology. ESRF provided beamtime (proposals MD-498 and MI-983, inhouse beamtimes IH-MI790 and BLC-6203). T.W. acknowledges support from the French research networks (RTRA) "Digiteo" and "Triangle de la Physique" (Grant Nos. 2009-79D and 2009-034T) and from the Agence nationale de la recherche (ANR; Grant ANR-EQPX-11-0031 "NanoimagesX"). F.P. acknowledges financial support through the DFG Cluster of Excellence
Munich-Center for Advanced Photonics (DFG EXC-158) and the European Research Council (FP7, Starting Grant 24012).

${ }^{1}$ A. Krauth, R. Blanc, A. Poveda, D. Jeanmonod, A. Morel, and G. Székely, "A mean three-dimensional atlas of the human thalamus: Generation from multiple histological data," NeuroImage 49, 2053-2062 (2010).

${ }^{2}$ G. Schulz, A. Morel, M. S. Imholz, H. Deyhle, T. Weitkamp, I. Zanette, F. Pfeiffer, C. David, M. Muller-Gerbl, and B. Müller, "Evaluating the microstructure of human brain tissues using synchrotron radiation-based micro computed tomography," Proc. SPIE 7804, 78040F (2010).

${ }^{3}$ M. Germann, A. Morel, F. Beckmann, A. Andronache, D. Jeanmonod, and B. Müller, "Strain fields in histological slices of brain tissue determined by synchrotron radiation-based micro computed tomography," J. Neurosci. Methods 170, 149-155 (2008).

${ }^{4}$ B. Müller, S. Lang, M. Dominietto, M. Rudin, G. Schulz, H. Deyhle, M. Germann, F. Pfeiffer, C. David, and T. Weitkamp, "High-resolution tomographic imaging of microvessels," Proc. SPIE 7078, 70780B (2008).

${ }^{5}$ S. Lang, M. Dominietto, and B. Müller, "Visualization of tumor vessels using synchrotron radiation-based micro computed tomography," J. Phys.: Conf. Ser. 186, 012088 (2009).

${ }^{6}$ M. D. Dominietto, "Multimodality approach to study the fractal physiology of tumor angiogenesis," Ph.D. dissertation (ETH Zurich, 2011).

${ }^{7}$ U. Bonse and M. Hart, "A x-ray interferometer," Appl. Phys. Lett. 6, 155-156 (1965).

${ }^{8}$ V. N. Ingal and E. A. Beliaevskaya, "X-ray plane-wave topography observation of the phase contrast from a non-crystalline object," J. Phys. D: Appl. Phys. 28, 2314 (1995).

${ }^{9}$ A. Snigirev, I. Snigireva, V. Kohn, S. Kuznetsov, and I. Schelokov, "On the possibilities of $\mathrm{x}$-ray phase contrast microimaging by coherent highenergy synchrotron radiation," Rev. Sci. Instrum. 66, 5486-5492 (1995).

${ }^{10}$ P. Cloetens, M. Pateyron-Salomé, J. Y. Buffière, G. Peix, J. Baruchel, F. Peyrin, and M. Schlenker, "Observation of microstructure and damage in materials by phase sensitive radiography and tomography," J. Appl. Phys. 81, 5878-5886 (1997).

${ }^{11}$ A. Momose, "Demonstration of X-ray Talbot interferometry," Jpn. J. Appl. Phys., Part 2 42, L866-L868 (2003).

${ }^{12}$ T. Weitkamp, A. Diaz, C. David, F. Pfeiffer, M. Stampanoni, P. Cloetens, and E. Ziegler, "X-ray phase imaging with a grating interferometer," Opt. Express 13, 6296-6304 (2005).

${ }^{13}$ B. Müller, G. Schulz, A. Mehlin, J. Herzen, S. Lang, M. Holme, I. Zanette, S. Hieber, H. Deyhle, F. Beckmann et al., "Grating-based tomography of human tissues," AIP Conf. Proc. 1466, 107-112 (2012).

${ }^{14}$ M. Holme, G. Schulz, H. Deyhle, T. Weitkamp, F. Beckmann, J. Lobrinus, F. Rikhtegar, V. Kurtcuoglu, I. Zanette, T. Saxer, and B. Müller, "Complementary $\mathrm{x}$-ray tomography techniques for histology-validated $3 \mathrm{~d}$ imaging of soft and hard human tissues using plaque-containing blood vessels as examples," Nat. Protoc. 9, 1401-1415 (2014).

${ }^{15}$ P. Cloetens, W. Ludwig, J. Baruchel, D. Van Dyck, J. Van Landuyt, J. Guigay, and M. Schlenker, "Holotomography: Quantitative phase tomography with micrometer resolution using hard synchrotron radiation x rays," Appl. Phys. Lett. 75, 2912-2914 (1999).

${ }^{16}$ M. Langer, P. Cloetens, and F. Peyrin, "Regularization of phase retrieval with phase-attenuation duality prior for 3-d holotomography," IEEE Trans. Image Process. 19, 2428-2436 (2010).

${ }^{17}$ M. Langer, A. Pacureanu, H. Suhonen, Q. Grimal, P. Cloetens, and F. Peyrin, "X-ray phase nanotomography resolves the $3 \mathrm{~d}$ human bone ultrastructure," PLoS One 7, e35691 (2012).

${ }^{18}$ D. Paganin, S. Mayo, T. Gureyev, P. Miller, and S. Wilkins, "Simultaneous phase and amplitude extraction from a single defocused image of a homogeneous object," J. Microsc. 206, 33-40 (2002).

${ }^{19}$ T. Gureyev, S. Mohammadi, Y. Nesterets, C. Dullin, and G. Tromba, "Accuracy and precision of reconstruction of complex refractive index in near-field single-distance propagation-based phase-contrast tomography," J. Appl. Phys. 114, 144906 (2013).

${ }^{20}$ J. Sun, P. Liu, S. Irvine, B. Pinzer, M. Stampanoni, and L. X. Xu, "Preliminary comparison of grating-based and in-line phase contrast X-ray imaging with synchrotron radiation for mouse kidney at tomcat," J. Instrum. 8, C06003 (2013).

${ }^{21}$ I. Zanette, S. Lang, A. Rack, M. Dominietto, M. Langer, F. Pfeiffer, T. Weitkamp, and B. Müller, "Holotomography versus X-ray grating interferometry: A quantitative study," Appl. Phys. Lett. 103, 244105 (2013). 
${ }^{22}$ P. C. Diemoz, A. Bravin, and P. Coan, "Theoretical comparison of three $\mathrm{X}$-ray phase-contrast imaging techniques: propagation-based imaging, analyzer-based imaging and grating interferometry," Opt. Express 20, 2789-2805 (2012).

${ }^{23}$ P. C. Diemoz, A. Bravin, M. Langer, and P. Coan, "Analytical and experimental determination of signal-to-noise ratio and figure of merit in three phase-contrast imaging techniques," Opt. Express 20, 27670-27690 (2012).

${ }^{24}$ T. Weitkamp, P. Tafforeau, E. Boller, P. Cloetens, J.-P. Valade, P. Bernard, F. Peyrin, W. Ludwig, L. Helfen, and J. Baruchel, "Status and evolution of the ESRF beamline ID19," AIP Conf. Proc. 1221, 33-38 (2010).

${ }^{25}$ J.-C. Labiche, O. Mathon, S. Pascarelli, M. A. Newton, G. G. Ferre, C. Curfs, G. Vaughan, A. Homs, and D. F. Carreiras, "The fast readout low noise camera as a versatile $\mathrm{x}$-ray detector for time resolved dispersive extended $\mathrm{x}$-ray absorption fine structure and diffraction studies of dynamic problems in materials science, chemistry, and catalysis," Rev. Sci. Instrum. 78, 091301 (2007).

${ }^{26}$ T. Weitkamp, I. Zanette, C. David, J. Baruchel, P. Bernard, M. Bech, H. Deyhle, T. Donath, J. Kenntner, S. Lang, J. Mohr, B. Müller, F. Pfeiffer, E. Reznikova, S. Rutishauser, G. Schulz, A. Tapfer, and J.-P. Valade, "Recent developments in X-ray Talbot interferometry at ESRF-ID19," Proc. SPIE 7804, 780406 (2010).

${ }^{27}$ I. Zanette, T. Weitkamp, S. Lang, M. Langer, J. Mohr, C. David, and J. Baruchel, "Quantitative phase and absorption tomography with an X-ray grating interferometer and synchrotron radiation," Phys. Status Solidi A 208, 2526-2532 (2011)

${ }^{28}$ S. Zabler, P. Cloetens, J. Guigay, J. Baruchel, and M. Schlenker, "Optimization of phase contrast imaging using hard x rays," Rev. Sci. Instrum. 76, 073705 (2005).

${ }^{29}$ J. Guigay, M. Langer, R. Boistel, and P. Cloetens, "Mixed transfer function and transport of intensity approach for phase retrieval in the Fresnel region," Opt. Lett. 32, 1617-1619 (2007).

${ }^{30}$ M. Langer, P. Cloetens, J. Guigay, and F. Peyrin, "Quantitative comparison of direct phase retrieval algorithms in in-line phase tomography," Med. Phys. 35, 4556-4566 (2008).

${ }^{31}$ M. Langer, P. Cloetens, B. Hesse, H. Suhonen, A. Pacureanu, K. Raum, and F. Peyrin, "Priors for X-ray in-line phase tomography of heterogeneous objects," Philos. Trans. R. Soc., A 372, 20130129 (2014).
${ }^{32}$ A. Groso, M. Stampanoni, R. Abela, P. Schneider, S. Linga, and R. Müller, "Phase contrast tomography: An alternative approach," Appl. Phys. Lett. 88, 214104 (2006).

${ }^{33}$ T. Weitkamp, D. Haas, D. Wegrzynek, and A. Rack, "ANKAphase: Software for single-distance phase retrieval from inline $\mathrm{x}$-ray phase-contrast radiographs," J. Synchrotron Radiat. 18, 617-629 (2011).

${ }^{34}$ I. Zanette, M. Bech, A. Rack, G. Le Duc, P. Tafforeau, C. David, J. Mohr, F. Pfeiffer, and T. Weitkamp, "Trimodal low-dose x-ray tomography," Proc. Natl. Acad. Sci. U.S.A. 109, 10199-10204 (2012).

${ }^{35}$ A. Mirone, E. Brun, E. Gouillart, P. Tafforeau, and J. Kieffer, "The pyhst2 hybrid distributed code for high speed tomographic reconstruction with iterative reconstruction and a priori knowledge capabilities," Nucl. Instrum. Methods Phys. Res., Sect. B 324, 41-48 (2014).

${ }^{36}$ F. Pfeiffer, C. Kottler, O. Bunk, and C. David, "Hard X-ray phase tomography with low-brilliance sources," Phys. Rev. Lett. 98, 108105 (2007).

${ }^{37}$ B. L. Henke, E. M. Gullikson, and J. C. David, "X-ray interactions: photoabsorption, scattering, transmission, and reflection at $E=50-30000 \mathrm{eV}$, $Z=1-92$," At. Data Nucl. Data Tables 54, 181-342 (1993).

${ }^{38}$ A. Andronache, M. von Siebenthal, G. Székely, and P. Cattin, "Non-rigid registration of multi-modal images using both mutual information and cross-correlation," Med. Image Anal. 12, 3-15 (2008).

${ }^{39}$ B. Müller, H. Deyhle, S. Lang, G. Schulz, T. Bormann, F. Fierz, and S. Hieber, "Three-dimensional registration of tomography data for quantification in biomaterials science," Int. J. Mater. Res. 103, 242-249 (2012).

${ }^{40}$ J. Herzen, T. Donath, F. Pfeiffer, O. Bunk, C. Padeste, F. Beckmann, A. Schreyer, and C. David, "Quantitative phase-contrast tomography of a liquid phantom using a conventional x-ray tube source," Opt. Express 17, 10010-10018 (2009).

${ }^{41}$ P. Modregger, D. Lübbert, P. Schäfer, and R. Köhler, "Spatial resolution in Bragg-magnified X-ray images as determined by Fourier analysis," Phys. Status Solidi A 204, 2746-2752 (2007).

${ }^{42}$ G. Schulz, T. Weitkamp, I. Zanette, F. Pfeiffer, F. Beckmann, C. David, S. Rutishauser, E. Reznikova, and B. Müller, "High-resolution tomographic imaging of a human cerebellum: Comparison of absorption and gratingbased phase contrast," J. R. Soc. Interface 7, 1665-1676 (2010).

${ }^{43} \mathrm{P}$. Thurner, F. Beckmann, and B. Müller, "An optimization procedure for spatial and density resolution in hard x-ray micro-computed tomography," Nucl. Instrum. Methods Phys. Res., Sect. B 225, 599-603 (2004). 